\title{
Coincident expression of $\beta$-catenin and cyclin D1 in endometrial stromal tumors and related high-grade sarcomas
}

\author{
Shuichi Kurihara ${ }^{1}$, Yoshinao Oda ${ }^{1}$, Yoshihiro Ohishi ${ }^{1}$, Eisuke Kaneki ${ }^{2}$, Hiroaki Kobayashi ${ }^{2}$, \\ Norio Wake ${ }^{2}$ and Masazumi Tsuneyoshi ${ }^{1}$ \\ ${ }^{1}$ Department of Anatomic Pathology, Graduate School of Medical Sciences, Kyushu University, Higashi-ku, \\ Fukuoka, Japan and ${ }^{2}$ Department of Obstetrics and Gynecology, Graduate School of Medical Sciences, \\ Kyushu University, Higashi-ku, Fukuoka, Japan
}

\begin{abstract}
Aberrant activation of the Wnt signaling pathway has been implicated in tumorigenesis of a wide range of tumors, including colorectal cancer. Regarding endometrial stromal tumors and related high-grade sarcomas, there have been some reports regarding nuclear accumulation of $\beta$-catenin. To clarify the function of the aberrant Wnt signaling pathway in these tumors, we searched for mutations of the CTNNB1 ( $\beta$-catenin) gene and $A P C$ gene by PCR direct sequencing and analyzed the methylation status of SFRP genes. We also examined overexpression of cyclin D1 and MMP-7, which are direct target genes of $\beta$-catenin. Eight endometrial stromal nodules, 16 low-grade endometrial stromal sarcomas, and 13 undifferentiated endometrial sarcomas were examined. PCR and direct sequencing revealed no mutation of the $\beta$-catenin gene or the APC gene. Concerning the promoter methylation status of SFRP genes, methylation-specific PCR revealed no significant difference between the group with nuclear $\beta$-catenin expression and that without nuclear $\beta$-catenin expression. Immunohistochemistry revealed overexpression of cyclin D1 in 2 out of 8 endometrial stromal nodules, 1 out of 17 low-grade endometrial stromal sarcomas, and 6 out of 13 undifferentiated endometrial sarcomas, and these 6 undifferentiated endometrial sarcomas simultaneously expressed nuclear $\beta$-catenin. Interestingly, all six undifferentiated endometrial sarcoma cases with cyclin D1 overexpression histologically featured rather uniform nuclei. In contrast, the six cases of undifferentiated endometrial sarcoma with highly pleomorphic nuclei were all negative for cyclin D1. In conclusion, among endometrial stromal tumors and related sarcomas, undifferentiated endometrial sarcomas featuring uniform nuclei were characterized by frequent coincident expression of $\beta$-catenin and cyclin D1. This finding raises the possibility that cyclin D1 is upregulated by $\beta$-catenin in these high-grade sarcomas previously called high-grade endometrial stromal sarcoma. Modern Pathology (2010) 23, 225-234; doi:10.1038/modpathol.2009.162; published online 6 November 2009
\end{abstract}

Keywords: endometrial stromal tumor; Wnt signaling pathway; cyclin D1; $\beta$-catenin

Endometrial stromal tumors are rare mesenchymal tumors of the uterine corpus and consist of benign endometrial stromal nodule and low-grade endometrial stromal sarcoma. ${ }^{1}$ On the basis of different cytological and biological features, high-grade endometrial sarcomas previously thought to consist of 'high-grade endometrial stromal sarcoma' and 'un-

Correspondence: Dr Y Oda, MD, PhD, Department of Anatomic Pathology, Graduate School of Medical Sciences, Kyushu University, 3-1-1 Maidashi, Higashi-ku, Fukuoka 812-8582, Japan.

E-mail: oda@surgpath.med.kyushu-u.ac.jp

Received 4 June 2009; revised 6 October 2009; accepted 7 October 2009; published online 6 November 2009 differentiated endometrial sarcoma' are now classified all together into 'undifferentiated endometrial sarcoma'. ${ }^{1}$ We have reported previously that among the current undifferentiated endometrial sarcomas, those previously labeled as 'high-grade endometrial stromal sarcoma' with monotonous cytological features share several molecular genetic and immunohistochemical characteristics with low-grade endometrial stromal sarcoma, whereas clearly pleomorphic undifferentiated endometrial sarcomas are considerably different from low-grade endometrial stromal sarcoma. ${ }^{2}$

Aberrant activation of the Wnt signaling pathway is known to contribute to tumorigenesis of a wide 
range of tumors, including colorectal cancer. ${ }^{3}$ The Wnt signaling pathway is regulated by many components such as APC and $\beta$-catenin, and genetic or epigenetic abnormalities of these components can cause dysregulated activation of the Wnt signaling pathway. These changes result in a maintained increase in the levels and transcriptional activity of $\beta$-catenin protein in the nucleus, with immunohistochemical results showing such an abnormal nuclear accumulation of $\beta$-catenin. ${ }^{4}$

Some kinds of mesenchymal tumors such as desmoid tumor and synovial sarcoma also frequently show nuclear accumulation of $\beta$-catenin. ${ }^{5,6}$ Regarding endometrial stromal sarcoma, $\mathrm{Ng}$ et $a l^{4}$ have reported that high-level nuclear $\beta$-catenin staining is seen in $40 \%$ of endometrial stromal sarcoma. We have also reported that $47 \%$ of lowgrade endometrial stromal sarcoma and $85 \%$ of undifferentiated endometrial sarcoma featuring uniform nuclei show nuclear $\beta$-catenin expression. ${ }^{2}$ From these findings, we speculated that the Wnt signaling pathway may be aberrantly activated in these tumors and contribute to tumorigenesis. However, genetic or epigenetic alterations responsible for nuclear $\beta$-catenin accumulation have remained obscure in endometrial stromal tumors. In addition, no reports are available regarding target genes induced by $\beta$-catenin in endometrial stromal tumors. Therefore, to clarify the contribution of the aberrant Wnt signaling pathway to the pathogenesis of endometrial stromal tumors and related highgrade sarcomas, we carried out a detailed examination of alterations in the pathway in this study.

Recent studies have shown that CCND1 (cyclin D1) and MMP-7 are important target genes induced by $\beta$-catenin. ${ }^{4,7-9}$ Cyclin D1 is known to stimulate the cell cycle and contribute to the process of cell proliferation, which is a characteristic of the stem cell phenotype. ${ }^{3}$ MMP-7 degrades extracellular matrices and is important in tumor invasion and metastasis. ${ }^{10,11}$ We investigated the frequency of cyclin D1 and MMP-7 overexpression by immunohistochemistry and attempted to determine whether they correlate with nuclear overexpression of $\beta$-catenin. The MIB-1 labeling index was used to assess the proliferative activity of the tumors.

A gain-of-function mutation of the CTNNB1 ( $\beta$-catenin) gene and a loss-of-function mutation of the $A P C$ gene have been reported to occur in many types of tumors. ${ }^{3}$ SFRP1, SFRP2, SFRP4, and SFRP5, which are also modulators of the Wnt signaling pathway, have been shown to be inhibited in several types of tumors by promoter hypermethylation. ${ }^{12-14}$ In low-grade endometrial stromal sarcomas and undifferentiated endometrial sarcomas, decreased expression of SFRP4 has been reported by Hrzenjak et $a .^{15}$ In relation to the Wnt signaling pathway, therefore, we analyzed gene mutations of $\beta$-catenin and $A P C$, and the methylation status of SFRP1, SFRP2, SFRP4, and SFRP5 genes in endometrial stromal tumor.

\section{Materials and methods}

\section{Patients and Tissue Specimens}

We searched for endometrial stromal tumors and related sarcomas in the files of the Department of Anatomic Pathology, Graduate School of Medical Sciences, Kyushu University, and retrieved 8 cases of endometrial stromal nodule, 16 cases of low-grade endometrial stromal sarcoma, and 13 cases of undifferentiated endometrial sarcoma. The histological diagnosis was made on light microscopic examination of hematoxylin-eosin-stained slides according to the recent WHO classification of tumors. ${ }^{1}$ These cases, other than the eight cases of endometrial stromal nodule, had been previously investigated in our study of immunohistochemical and molecular genetic analyses. ${ }^{2}$ As we reported previously, 16 low-grade endometrial stromal sarcomas were mainly composed of uniform tumor cells resembling normal endometrial stromal cells with uniform nuclei showing minimal cytological atypia (Figure 1b). Of the 13 undifferentiated endometrial sarcoma, 7 featured rather monotonous cytological uniformity reminiscent of low-grade endometrial stromal sarcoma, but they were separated from lowgrade endometrial stromal sarcoma because they showed significant nuclear atypia (Figure 1c). When minor nuclear pleomorphism with nucleomegaly, nuclear hyperchromatism, and prominent nucleoli were present singly or in combination in a tumor, it was regarded as having significant atypia. These seven cases of undifferentiated endometrial sarcoma were considered to be identical to the tumors previously called 'high-grade endometrial stromal sarcoma' in the AFIP atlas of tumor pathology. ${ }^{16}$ The other six cases of undifferentiated endometrial sarcoma were typical ones composed of highly pleomorphic tumor cells (Figure 1d). As in our previous study, we call the former undifferentiated endometrial sarcoma group 'undifferentiated endometrial sarcoma with nuclear uniformity' and the latter group 'undifferentiated endometrial sarcoma with nuclear pleomorphism'. ${ }^{2}$

\section{Immunohistochemistry}

Representative formalin-fixed, paraffin-embedded materials for immunohistochemical studies were available in all 37 cases. The primary monoclonal antibodies used were as follows: $\beta$-catenin (clone 14, dilution 1:200; Transduction Laboratories, Lexington, KY, USA), cyclin D1 (clone P2D11F11, dilution 1:25; Novocastra, New Castle, UK), MMP-7 (clone 141-7B2, dilution 1:150; Daiichi Fine Chemical, Takaoka, Japan), and Ki-67 (clone MIB-1, dilution 1:100; Dako, Grostrup, Denmark). Sections (3- $\mu \mathrm{m}-$ thick) were deparaffinized and rehydrated through xylene and ethanol. Endogenous peroxidase activity was then blocked by methanol containing $0.3 \%$ hydrogen peroxidase for $30 \mathrm{~min}$. Antigen retrieval 

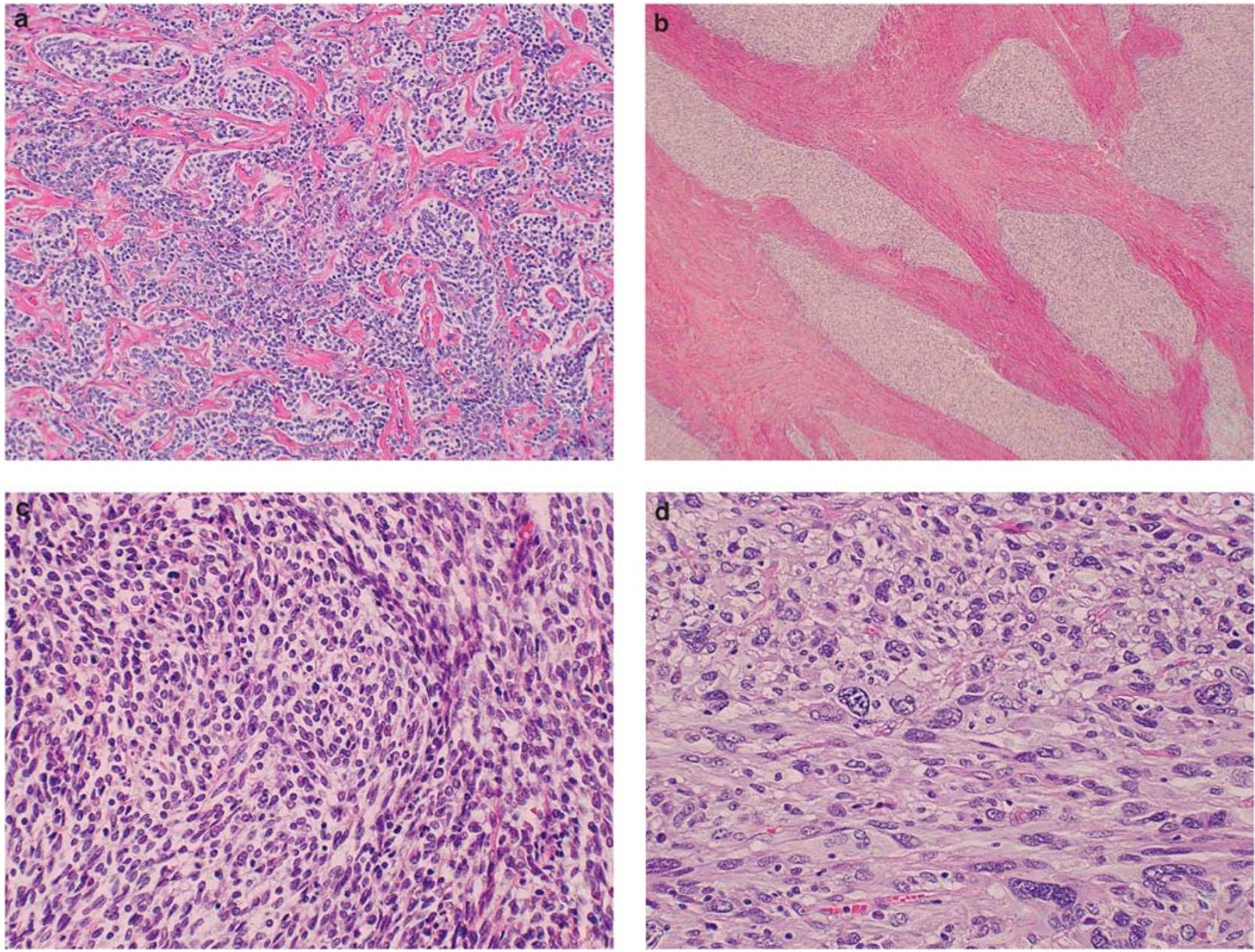

Figure 1 (a) Endometrial stromal nodule with sex cord-like differentiation (case 8). (b) Low-grade endometrial stromal sarcoma (case 23). (c) Undifferentiated endometrial sarcoma with nuclear uniformity (case 28). (d) Undifferentiated endometrial sarcoma with nuclear pleomorphism (case 35).

was performed by heating tissue sections at $100^{\circ} \mathrm{C}$ in $0.01 \mathrm{~mol} / \mathrm{l}$ sodium citrate buffer and $0.01 \%$ Tween $(\mathrm{pH}$ 6.0) ( $\beta$-catenin and Ki-67) or in $0.01 \mathrm{~mol} / \mathrm{l}$ sodium citrate buffer ( $\mathrm{pH}$ 6.0) (cyclin D1 and MMP-7) for $20 \mathrm{~min}$ in a microwave oven. The sections were incubated with primary antibodies at $4{ }^{\circ} \mathrm{C}$ overnight, followed by staining with a streptavidin-biotin-peroxidase kit (Nichirei, Tokyo, Japan). For evaluating $\beta$-catenin, cyclin D1, and MMP-7, the percentage of stained tumor cells was assessed to the nearest $10 \%$ (eg, 10-30\%). For those cases in which positive tumor cells consisted of $5 \%$ or less of total tumor cells, the percentage was recorded as $0 \%$, less than $1 \%$, or $1-5 \%$. For evaluating $\beta$-catenin staining, sections were considered to show overexpression if $30 \%$ or more of the tumor cells showed nuclear staining. ${ }^{4}$ As for cyclin D1, staining of more than $5 \%$ of the tumor nuclei was needed for a case to be scored positive. ${ }^{6}$ The MIB-1 labeling index, which refers to a percentage of positive tumor cells, was determined by counting at least 500 tumor cells.

\section{Mutational Analysis of $\beta$-Catenin and APC Genes}

Mutation analysis of the $\beta$-catenin and $A P C$ genes was performed on the same 37 samples. Genomic DNA from formalin-fixed, paraffin-embedded tissue was extracted using the standard proteinase $\mathrm{K}$ digestion and phenol/chloroform extraction methods. The PCR was performed for the entire region of exon 3 of the $\beta$-catenin gene and the mutation cluster region of the $A P C$ gene exon 15 from codons 1274 to 1523 . The set of primers used for $\beta$-catenin gene was the same as that previously described. ${ }^{17}$ As for the $A P C$ gene, seven overlapping sets of primers were used. The primer sets designed by Yagi et $a l^{18}$ were used, except for the sixth primer set. In place of it, we used a forward primer $5^{\prime}$-agctcaaaccaagcga gaag- $3^{\prime}$ and a reverse primer $5^{\prime}$-ctttccgtggcaaaatgtaa$3^{\prime}$, which generated a PCR product of $150 \mathrm{bp}$. PCR was performed in a final reaction volume of $20 \mu \mathrm{l}$ containing $100 \mathrm{ng}$ of template DNA, $1.5 \mathrm{mM} \mathrm{MgCl}_{2}$, $1 \times$ PCR buffer (Applied Biosystems, Foster City, CA, USA), $0.25 \mathrm{mM}$ dNTP mix, $0.5 \mu \mathrm{M}$ each of sense 
and antisense primer, and $1 \mathrm{U}$ of Gold Taq polymerase (Applied Biosystems). Human genomic DNA (Clonetech, Palo Alto, CA, USA) was used as a positive control for each PCR. The PCR products were electrophoresed through $2.0 \%$ agarose gel with ethidium bromide to confirm the correct amplification. The correctly amplified products were then purified by centrifugal filter devices of Microcon (Millipore, Bedford, MA, USA). After purification, direct sequencing was carried out by the dideoxy chain-termination method using the BigDye Terminator and the PerkinElmer ABI Prism 310 sequence analyzer (Applied Biosystems).

\section{Methylation-Specific PCR for the Promoter Regions of SFRP Genes}

Bisulfite conversion was performed with $1 \mu \mathrm{g}$ of genomic DNA using the reagents provided with the CpGenome DNA Modification Kit (InterGen, Burlington, MA, USA). Methylation-specific PCR was performed to determine the DNA methylation status of CpG islands of the SFRP promoter regions. The primer pairs used in this study were the same as those previously described in the literature. ${ }^{19}$ Bisulfite-treated human genomic DNA (Clonetech) and CpGenome Universal Methylated DNA (Chemicon, Temecula, CA, USA) were used as positive controls for unmethylation and methylation, respectively.

\section{RT-PCR Analysis for the JAZF1-JJAZ1 Fusion Gene}

Total RNA extraction and reverse transcription-PCR (RT-PCR) of the 37 samples and direct sequencing for positive cases were performed as previously described. ${ }^{2}$ All these cases, including eight cases of endometrial stromal nodule, were examined at the same time in the previous study, but endometrial stromal nodules were not reported because benign endometrial stromal nodule was outside the scope of that study. Assessment of RNA quality by realtime quantitative RT-PCR for glyceraldehyde-3phosphate dehydrogenase (GAPDH) was also performed at the same time, as previously described. ${ }^{2}$

\section{Results}

\section{Morphological Findings}

Among eight cases of endometrial stromal nodules, two cases (cases 3 and 8) showed focal sex cord-like differentiation (Figure 1a). Morphological findings regarding 16 cases of low-grade endometrial stromal sarcoma and 13 cases of undifferentiated endometrial sarcomas were reported in the previous study (Figure $1 \mathrm{~b}-\mathrm{d}$ ). ${ }^{2}$ A primary pelvic tumor arising from uterine serosa associated with endometriosis (case 10) was included in the low-grade endometrial stromal sarcoma group.

\section{Immunohistochemistry}

Table 1 summarizes the immunohistochemical results. Three $(37 \%)$ out of 8 cases of endometrial stromal nodule, $8(50 \%)$ out of 16 low-grade endometrial stromal sarcoma, $6(85 \%)$ out of 7 undifferentiated endometrial sarcoma with nuclear uniformity, and $2(33 \%)$ out of 6 undifferentiated endometrial sarcoma with nuclear pleomorphism were judged as showing overexpression of $\beta$-catenin. We have already reported these results concerning low-grade endometrial stromal sarcoma, undifferentiated endometrial sarcoma with nuclear uniformity, and undifferentiated endometrial sarcoma with nuclear pleomorphism in the previous study. $^{2}$ The nuclear expression of cyclin D1 was assessed as positive in two cases $(25 \%)$ of endometrial stromal nodule, in one case $(5 \%)$ of low-grade endometrial stromal sarcoma, in six cases $(85 \%)$ of undifferentiated endometrial sarcoma with nuclear uniformity, and none of undifferentiated endometrial sarcoma with nuclear pleomorphism. All the six cases of undifferentiated endometrial sarcoma with nuclear uniformity that were positive for cyclin D1 also showed overexpression of $\beta$-catenin (Figure 2a and b). The frequency of cyclin D1 overexpression was significantly higher in undifferentiated endometrial sarcoma with nuclear uniformity (85\%) compared with that in endometrial stromal nodule $(5 \% ; P=0.031)$, low-grade endometrial stromal sarcoma $(5 \% ; P<0.001)$, and undifferentiated endometrial sarcoma with nuclear pleomorphism $(0 \% ; P=0.004)$ (Fisher's exact probability test). Among the 13 combined cases of undifferentiated endometrial sarcoma with nuclear uniformity and undifferentiated endometrial sarcoma with nuclear pleomorphism, the frequency of cyclin D1 overexpression was significantly higher in the $\beta$-catenin-positive tumors compared with those without overexpression of $\beta$-catenin $(P<0.05$, Fisher's exact probability test); however, there was no statistically significant correlation between $\beta$-catenin and cyclin D1 overexpression in each histological group (Fisher's exact probability test) (Table 2). Staining of MMP-7 was detected in the cytoplasms of tumor cells in three endometrial stromal nodules $(38 \%)$ and three low-grade endometrial stromal sarcomas. $(19 \%)$, but was not detected in undifferentiated endometrial sarcoma (Figure 2c). Of these six positive cases, two endometrial stromal nodules (cases 6 and 8) showed only focal and weak staining of MMP-7. One endometrial stromal nodule (case 5) and three low-grade endometrial stromal sarcomas featured diffuse staining ranging from 20 to $50 \%$ of tumor cells. Among the 24 combined cases of endometrial stromal nodules and low-grade endometrial stromal sarcomas, there was no significant correlation between $\beta$-catenin overexpression and expression of MMP-7 (Fisher's exact probability test). Concerning the MIB-1 labeling index, there was no significant difference between endometrial 
Table 1 Results of immunohistochemistry

\begin{tabular}{|c|c|c|c|c|c|c|c|}
\hline \multirow[b]{2}{*}{ Case no. } & \multirow[b]{2}{*}{ Histology } & \multirow[b]{2}{*}{ Age } & \multirow{2}{*}{$\begin{array}{c}R T-P C R \\
\text { JAZF1-JJAZ1 }\end{array}$} & \multicolumn{4}{|c|}{ Immunohistochemistry (\%) } \\
\hline & & & & $\beta$-Catenin & Cyclin D1 & $M M P-7$ & $M I B-1$ \\
\hline 1 & ESN & 51 & $(+)$ & 0 & 0 & 0 & 0.5 \\
\hline 2 & ESN & 49 & NA & 0 & 0 & 0 & 1.6 \\
\hline 3 & ESN & 41 & $(-)$ & 70 & 0 & 0 & 2.8 \\
\hline 4 & ESN & 80 & $(+)$ & 40 & 0 & 0 & 7.5 \\
\hline 5 & ESN & 49 & $(+)$ & 0 & 40 & 20 & 0.5 \\
\hline 6 & ESN & 40 & NA & $<1$ & 0 & $<1$ & 0.2 \\
\hline 7 & ESN & 48 & $(-)$ & 0 & 0 & 0 & 0.4 \\
\hline 8 & ESN & 43 & $(+)$ & 50 & 40 & $<1$ & 7.8 \\
\hline 9 & ESS-LG & NA & NA & 30 & 0 & 0 & 2.5 \\
\hline 10 & ESS-LG & 41 & NA & 0 & 0 & 0 & 0.1 \\
\hline 11 & ESS-LG & 29 & $(-)$ & 50 & 0 & 0 & 1.0 \\
\hline 12 & ESS-LG & 34 & NA & 70 & 0 & 0 & 2.8 \\
\hline 13 & ESS-LG & 43 & NA & 40 & 0 & 0 & 1.2 \\
\hline 14 & ESS-LG & 35 & NA & 0 & 0 & 0 & 1.1 \\
\hline 15 & ESS-LG & 45 & $(-)$ & 0 & 0 & 50 & 0.7 \\
\hline 16 & ESS-LG & 51 & $(+)$ & 80 & $<1$ & 0 & 4.4 \\
\hline 17 & ESS-LG & 34 & $(-)$ & 30 & 1 & 0 & 6.3 \\
\hline 18 & ESS-LG & 34 & $(-)$ & 0 & 0 & 0 & 0.4 \\
\hline 19 & ESS-LG & 41 & $(-)$ & 0 & 0 & 0 & 2.2 \\
\hline 20 & ESS-LG & 47 & $(+)$ & 0 & 0 & 40 & 0.1 \\
\hline 21 & ESS-LG & 41 & $(-)$ & 0 & 0 & 0 & 1.5 \\
\hline 22 & ESS-LG & 47 & $(+)$ & $<1$ & 0 & 0 & 0.1 \\
\hline 23 & ESS-LG & 49 & $(+)$ & 70 & 50 & 50 & 3.3 \\
\hline 24 & ESS-LG & 47 & $(+)$ & 40 & 0 & 0 & 0.6 \\
\hline 25 & UES-U & 24 & NA & 40 & 70 & 0 & 20.3 \\
\hline 26 & UES-U & 23 & NA & 30 & 30 & 0 & 6.6 \\
\hline 27 & UES-U & 50 & NA & 40 & 70 & 0 & 8.5 \\
\hline 28 & UES-U & 34 & $(-)$ & 70 & 60 & 0 & 53.9 \\
\hline 29 & UES-U & 49 & NA & 70 & 30 & 0 & 27.5 \\
\hline 30 & UES-U & 53 & $(-)$ & 50 & 70 & 0 & 6.9 \\
\hline 31 & UES-U & 69 & $(+)$ & $<1$ & $<1$ & 0 & 9.7 \\
\hline 32 & UES-P & 60 & NA & 40 & 0 & 0 & 20.8 \\
\hline 33 & UES-P & 64 & NA & $1-5$ & 0 & 0 & 8.4 \\
\hline 34 & UES-P & 56 & $(-)$ & 40 & 0 & 0 & 28.3 \\
\hline 35 & UES-P & 54 & NA & 0 & 0 & 0 & 31.4 \\
\hline 36 & UES-P & 67 & $(-)$ & 0 & $<1$ & 0 & 12.7 \\
\hline 37 & UES-P & 56 & $(-)$ & 0 & 0 & 0 & 26.2 \\
\hline
\end{tabular}

ESN, endometrial stromal nodule; ESS-LG, low-grade endometrial stromal sarcoma; NA, not available; UES-P, undifferentiated endometrial sarcoma with nuclear pleomorphism; UES-U, undifferentiated endometrial sarcoma with nuclear uniformity.

stromal nodule (mean 2.6) and low-grade endometrial stromal sarcoma (mean 1.7) (Figure 2d). MIB-1 labeling indices of undifferentiated endometrial sarcoma with nuclear uniformity (mean 19.0) and undifferentiated endometrial sarcoma with nuclear pleomorphism (mean 21.3) were both higher than that of low-grade endometrial stromal sarcoma $(P<0.05$, Mann-Whitney $U$-test $)$. Among the 24 combined cases of endometrial stromal nodules and low-grade endometrial stromal sarcomas, the MIB-1 labeling index of the $\beta$-catenin-positive group was significantly higher than that of the $\beta$-cateninnegative group $(P<0.05$, Mann-Whitney $U$-test $)$.

\section{Mutational Analysis of the $\beta$-Catenin and APC Genes}

No mutated sequence of the $\beta$-catenin and $A P C$ genes was detected in the 37 samples.

\section{Methylation-Specific PCR for the Promoter Regions of SFRP Genes}

Figure 3a reveals the results of MSP for SFRP1, SFRP2, SFRP4, and SFRP5. Figure 3b shows the representative PCR products in the examined genes. Among the tumors with overexpression of $\beta$-catenin, SFRP1 promoter hypermethylation was detected in two $(22 \%)$ out of nine cases. Regarding SFRP2, SFRP4, and SFRP5, the tumors were positive for promoter hypermethylation in $6(40 \%)$ out of 15 cases, $2(11 \%)$ out of 19 cases, and $9(60 \%)$ out of 15 cases, respectively. In contrast, among the tumors without overexpression of $\beta$-catenin, promoter hypermethylation of SFRP1, SFRP2, SFRP4, and SFRP5 was observed in 0 out of 9 cases, $8(47 \%)$ out of 17 cases, $1(<1 \%)$ out of 18 cases, and $6(38 \%)$ out of 16 cases, respectively. The frequencies of promoter hypermethylation of SFRP1,SFRP2, SFRP4, and 

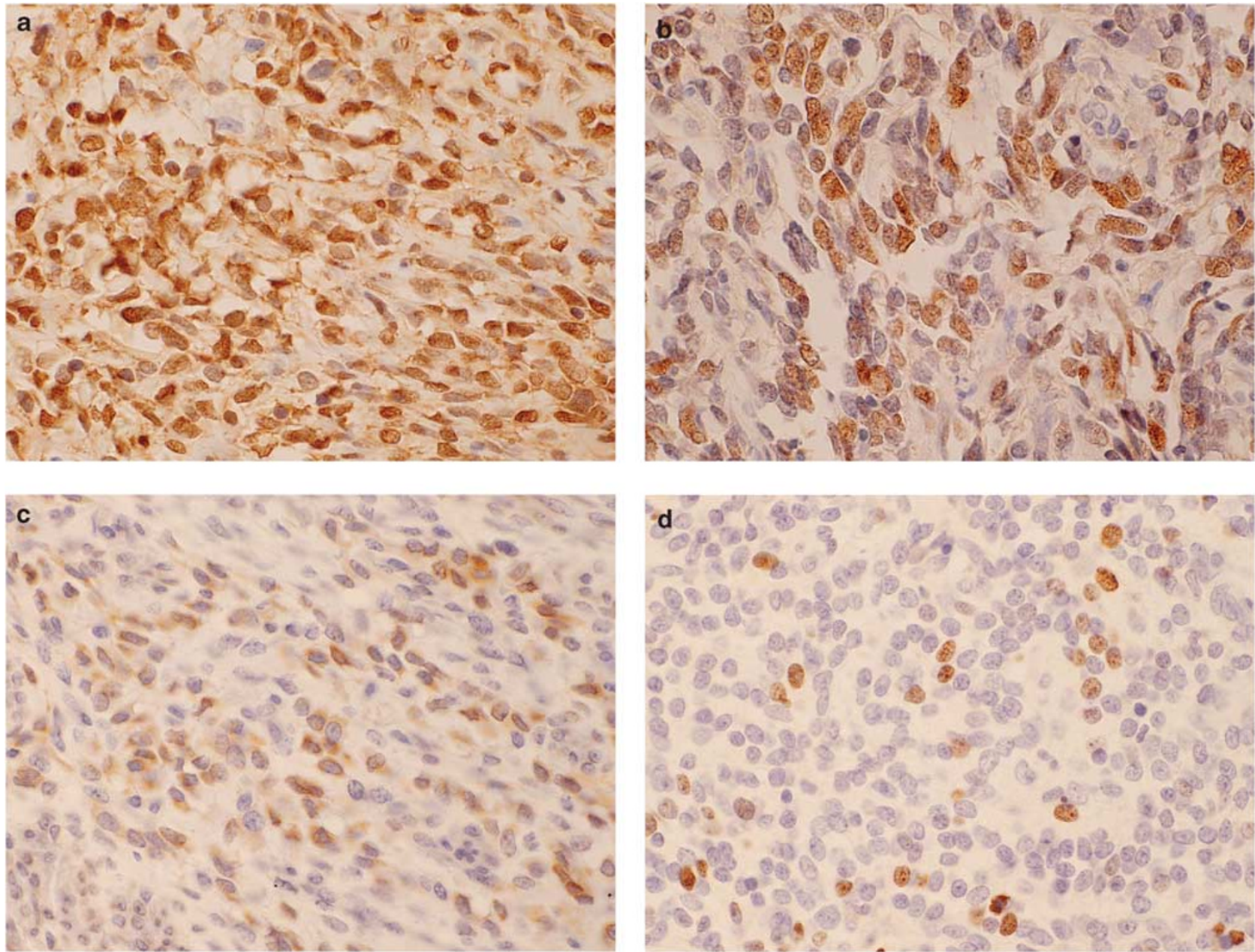

Figure 2 (a and b) Immunohistochemistry of $\beta$-catenin (a) and cyclin D1 (b) in a case of undifferentiated endometrial sarcoma (case 28). Widespread nuclear staining is observed in both $\beta$-catenin (60\%) and cyclin D1 (70\%) immunostaining. (c) MMP-7 immunostaining in a case of low-grade endometrial stromal sarcoma (case 23; 50\%). (d) MIB-1 immunostaining in a case of endometrial stromal nodule (case $8 ; 7.8 \%$ )

Table 2 Results of $\beta$-catenin and cyclin D1 expression in each histological group

\begin{tabular}{|c|c|c|c|c|c|}
\hline \multirow[t]{2}{*}{ Histology } & \multicolumn{2}{|c|}{$\beta$-Catenin (+) } & \multicolumn{2}{|c|}{$\beta$-Catenin (-) } & \multirow[t]{2}{*}{ Correlation } \\
\hline & & $\mathrm{n}$ & & $\mathrm{n}$ & \\
\hline \multirow[t]{2}{*}{$\operatorname{ESN}(n=8)$} & Cyclin D1 (+) & 1 & Cyclin D1 (+) & 1 & \multirow{2}{*}{ NS $(P>0.05)$} \\
\hline & Cyclin D1 (-) & 2 & Cyclin D1 (-) & 4 & \\
\hline \multirow{2}{*}{ ESS-LG $(n=16)$} & Cyclin D1 (+) & 1 & Cyclin D1 (+) & 0 & \multirow[t]{2}{*}{ NS $(P>0.05)$} \\
\hline & Cyclin D1 (-) & 7 & Cyclin D1 (-) & 8 & \\
\hline \multirow[t]{2}{*}{ UES-U $(n=7)$} & Cyclin D1 (+) & 6 & Cyclin D1 (+) & 0 & \multirow{2}{*}{ NS $(P>0.05)$} \\
\hline & Cyclin D1 (-) & 0 & Cyclin D1 (-) & 1 & \\
\hline \multirow[t]{2}{*}{ UES-P $(n=6)$} & Cyclin D1 (+) & 0 & Cyclin D1 (+) & 0 & \multirow[t]{2}{*}{ NS $(P>0.05)$} \\
\hline & Cyclin D1 (-) & 2 & Cyclin D1 (-) & 4 & \\
\hline \multirow[t]{2}{*}{ UES-U+UES-P $(n=13)$} & Cyclin D1 (+) & 6 & Cyclin D1 (+) & 0 & \multirow[t]{2}{*}{ Significant $(P=0.02)$} \\
\hline & Cyclin D1 (-) & 2 & Cyclin D1 (-) & 5 & \\
\hline
\end{tabular}

ESN, endometrial stromal nodule; ESS-LG, low-grade endometrial stromal sarcoma; NS, not significant; UES-P, undifferentiated endometrial sarcoma with nuclear pleomorphism; UES-U, undifferentiated endometrial sarcoma with nuclear uniformity. 

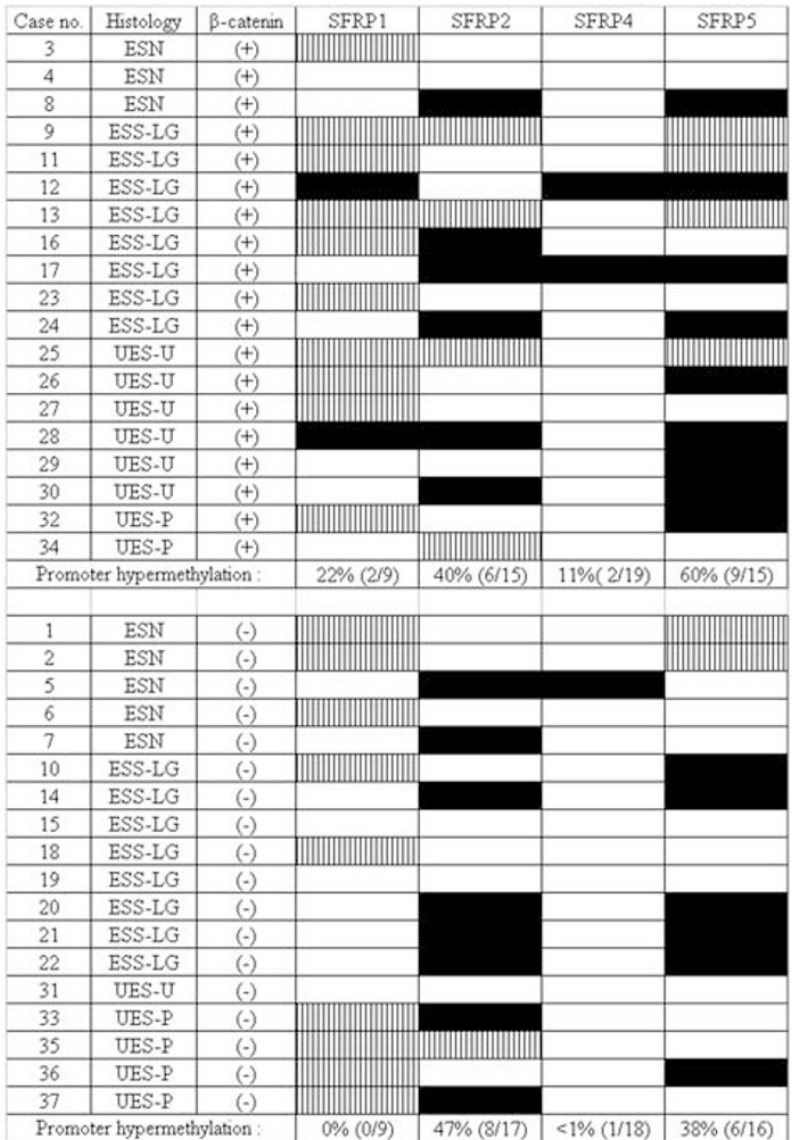

b

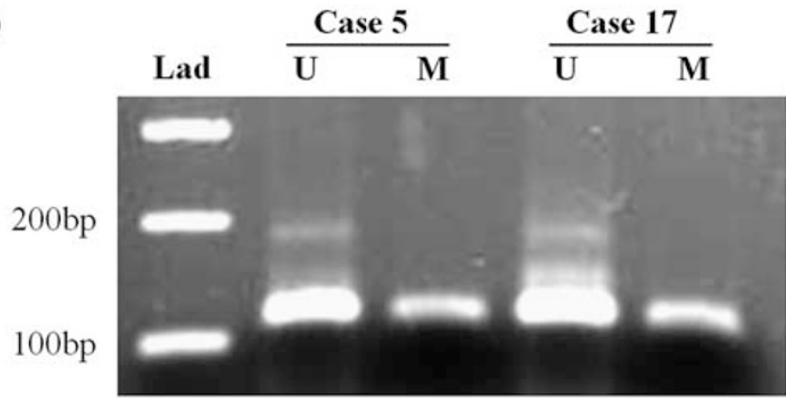

Figure 3 (a) Methylation profiles of four types of SFRP genes in the $\beta$-catenin-positive group (upper half) and the $\beta$-cateninnegative group (lower half). A filled box (black box) indicates that promoter hypermethylation was detected by methylationspecific PCR, and an open box (white box) indicates that no methylation was detected. Adequate results were not available in vertical-striped boxes. (b) An example of MSP for the SFRP4 gene. Cases 5 and 17 show hypermethylation of the SFRP4 gene promoter. PCR products were amplified by unmethylated (U) and methylated (M) specific primers.

SFRP5 were not significantly different between the $\beta$-catenin-positive and $\beta$-catenin-negative groups.

\section{RT-PCR Analysis for the JAZF1-JJAZ1 Fusion Gene}

We have previously reported the manner of determining noninformative samples and the results concerning the cases of low-grade endometrial stromal sarcoma and undifferentiated endometrial sarcoma. ${ }^{2}$ Fourteen cases were judged as noninformative because real-time RT-PCR revealed that $G A P D H$ expression was lower than the cutoff level. Among the 23 informative cases, we detected the JAZF1-JJAZ1 fusion transcript in $4(67 \%)$ out of 6 cases of endometrial stromal nodule, 5 (45\%) out of 12 cases of low-grade endometrial stromal sarcoma, $1(33 \%)$ out of 3 cases of undifferentiated endometrial sarcoma with nuclear uniformity, and 0 out of 3 cases of undifferentiated endometrial sarcoma with nuclear pleomorphism (Table 1). There was no statistically significant correlation between positive JAZF1-JJAZ1 fusion and nuclear overexpression of $\beta$-catenin (Fisher's exact probability test). The sequence was proved by direct sequencing, and a breakpoint identical to that described previously ${ }^{20}$ was detected in all of these 10 cases.

\section{Discussion}

In this study, we found that coincident overexpression of $\beta$-catenin and cyclin D1 is frequent in undifferentiated endometrial sarcoma with nuclear uniformity. In contrast, coincident overexpression of $\beta$-catenin and cyclin D1 was found to be rare among endometrial stromal nodules, low-grade endometrial stromal sarcomas, and undifferentiated endometrial sarcoma with nuclear pleomorphism. We have previously speculated that the morphological difference between undifferentiated endometrial sarcoma with nuclear uniformity and undifferentiated endometrial sarcoma with nuclear pleomorphism, which are both biologically high-grade lesions, may be derived from different histogenetic mechanisms based on the different prevalence of several molecular genetic and immunohistochemical findings. ${ }^{2}$ In this study, undifferentiated endometrial sarcoma with nuclear pleomorphism, in contrast to undifferentiated endometrial sarcoma with nuclear uniformity, did not show coincident overexpression of $\beta$-catenin and cyclin D1. This finding may support our speculation that undifferentiated endometrial sarcoma with nuclear uniformity and undifferentiated endometrial sarcoma with nuclear pleomorphism may represent two separate entities. In addition, positive immunoreactivity for cyclin D1 may be helpful in differential diagnosis between low-grade endometrial stromal sarcoma and undifferentiated endometrial sarcoma with nuclear uniformity.

Cyclin D1 is frequently overexpressed in human cancers of diverse histological origin, and its gene is known as a protooncogene. ${ }^{21}$ Several mechanisms resulting in cyclin D1 overexpression have been clarified such as chromosomal translocation in mantle cell lymphoma and gene amplification in breast cancer. ${ }^{21}$ It has also been clarified that 
increased transcriptional activity of cyclin $D 1$ gene is regulated by intracellular signaling events, leading to activation of various transcription factors. Tetsu et $a l^{7}$ have reported that $\beta$-catenin regulates expression of cyclin D1 in colon carcinoma cells, and coincident overexpression of $\beta$-catenin and cyclin D1 has been reported in several kinds of tumors, such as colorectal cancer, ${ }^{22}$ breast cancer, ${ }^{23}$ thyroid papillary carcinoma, ${ }^{24}$ and desmoid tumor. ${ }^{6}$ Our results raise the possibility that cyclin D1 may be upregulated through an activated $\mathrm{Wnt} / \beta$-catenin pathway in undifferentiated endometrial sarcoma with nuclear uniformity. We consider that further studies to support this speculation would be of value because the Wnt signaling pathway and cyclin D1 could potentially be a future target of anticancer drugs. ${ }^{3,25}$

Expression of MMP-7, which is another target of $\beta$-catenin, was limited in this study to only one case of endometrial stromal nodule and three of lowgrade endometrial stromal sarcoma, and there was no significant correlation between $\beta$-catenin overexpression and MMP-7 expression. MMP-7 did not seem to have a crucial function in tissue invasion and metastasis in low-grade endometrial stromal sarcoma and undifferentiated endometrial sarcoma in this study. Concerning the MIB-1 labeling index, there was no significant difference between endometrial stromal nodule and low-grade endometrial stromal sarcoma in this study. This result suggests the same degree of proliferative activity in both groups. The mechanisms making the different phenotypes between endometrial stromal nodule and low-grade endometrial stromal sarcoma remain unclear.

The Wnt signaling pathway can be activated by changes occurring at any level of the pathway, and many alterations other than mutations of $A P C$ and $\beta$-catenin have been reported. ${ }^{3}$ We could not detect any mutations of the $\beta$-catenin and $A P C$ genes among the cases showing overexpression of $\beta$-catenin. It is therefore possible that another mechanism of $\beta$-catenin accumulation may be present in these tumors. As for the $\beta$-catenin gene, Jung et $a l^{26}$ have made similar observations. They have reported finding only one endometrial stromal nodule out of 15 endometrial stromal tumors (2 endometrial stromal nodule, 7 low-grade endometrial stromal sarcoma, and 6 undifferentiated endometrial sarcoma) to have a $\beta$-catenin mutation. ${ }^{26}$ Hrzenjak et al have reported that in endometrial stromal sarcomas, the SFRP4 mRNA concentrations are decreased, being lower in undifferentiated endometrial sarcoma than in low-grade endometrial stromal sarcoma. We could not support their observation, as our results suggest that promoter hypermethylation of SFRP4 is rare in endometrial stromal tumors and undifferentiated endometrial sarcoma. Mechanisms other than promoter hypermethylation, which suppresses expression of SFRP4, may therefore be present in these tumors.
As Micci et $a l^{27}$ stated in their review article, it is unknown whether interference with the Wnt system and the JAZF1 occurs in the same tumors or if these are alternative pathogenetic mechanisms. In this study, the coexistence of JAZF1-JJAZ1 fusion and nuclear overexpression of $\beta$-catenin was observed in five cases, and there was no statistically significant correlation between them. However, definitive results regarding whether there is a correlation are not yet available due to our limited number of cases. In this study, we have presented data regarding the prevalence of JAZF1-JJAZ1 among the benign endometrial stromal nodules, in addition to the previously reported data on low-grade endometrial stromal sarcoma and undifferentiated endometrial sarcoma. ${ }^{2}$ Our results showing a high prevalence of JAZF1-JJAZ1 among endometrial stromal nodules $(66 \%)$ are consistent with the several reports dealing with endometrial stromal nodule cases. Regarding endometrial stromal nodule, JAZF1-JJAZ1 gene fusion was detected in all three cases reported by Koontz et $a{ }^{20}{ }^{20}$ in the two cases reported by Huang et $a{ }^{31}{ }^{31}$ and in all four cases reported by Nucci et al. ${ }^{28}$ As for endometrial stromal tumors with sex cordlike differentiation, there have been two cases reported in the literature in which JAZF1-JJAZ1 fusion was detected..$^{29,30}$ In our study, one of the two cases of endometrial stromal nodule with sex cordlike differentiation was positive for the JAZF1-JJAZ1 fusion transcript, supporting the idea that genetic heterogeneity exists among endometrial stromal tumors with variant histology. ${ }^{31}$

On the basis of review of the literature and our study, we have hypothesized a simplified model for the histogenesis of endometrial stromal tumors and related high-grade sarcomas (Figure 4). It is commonly accepted that endometrial stromal nodule and low-grade endometrial stromal sarcoma belong to the same category featuring endometrial stromal differentiation. We reported in the previous study that undifferentiated endometrial sarcoma with nuclear uniformity features similar immunophenotype with low-grade endometrial stromal sarcoma, such as positive staining for estrogen receptor/ progesterone receptor and $\beta$-catenin and negative staining for $\mathrm{p}^{2}$. $^{2}$ Accordingly, we speculated that undifferentiated endometrial sarcoma with nuclear uniformity may belong to the same category with low-grade endometrial stromal sarcoma. This study suggested that cyclin D1 immunoreactivity may be a characteristic immunophenotype of undifferentiated endometrial sarcoma with nuclear uniformity, which may contribute to the high-grade malignancy of the tumor. It is unclear whether undifferentiated endometrial sarcoma with nuclear uniformity is 'high-grade' endometrial stromal sarcoma arising de novo, or 'high-stage' endometrial stromal sarcoma developing from low-grade endometrial stromal sarcoma. It may be that both of these occur. In contrast, undifferentiated endometrial sarcoma with nuclear pleomorphism may be considered as a truly 


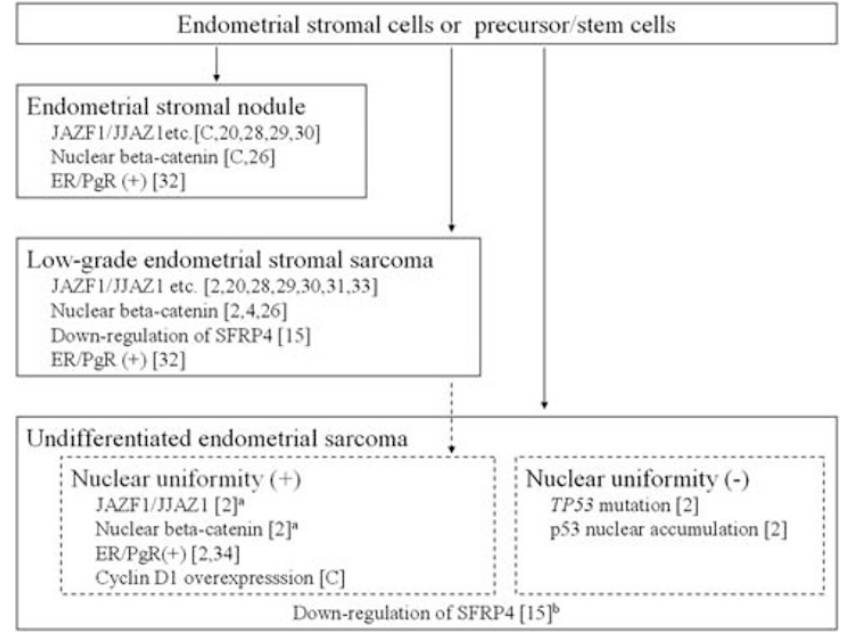

Figure 4 Schema of hypothesis for histogenesis of endometrial stromal tumors and related high-grade sarcomas. The numbers in parentheses indicate those of references. The letter ' $\mathrm{C}$ ' in parentheses indicates this study. ${ }^{a}$ These features concerning undifferentiated endometrial sarcoma are reported in other articles without separating undifferentiated endometrial sarcoma into two groups. ${ }^{15,20}$ bThis feature in undifferentiated endometrial sarcoma are reported without separating undifferentiated endometrial sarcoma into two groups. ${ }^{2}$

'undifferentiated' sarcoma showing no evidence of endometrial stromal differentiation. In addition, our study suggested that positive cyclin D1 immunoreactivity may be another feature distinguishing undifferentiated endometrial sarcoma with nuclear uniformity from undifferentiated endometrial sarcoma with nuclear pleomorphism, the latter of which is characterized by frequent p53 abnormality.

In conclusion, this study suggested that among endometrial stromal tumors and related high-grade sarcomas, only undifferentiated endometrial sarcoma featuring uniform nuclei frequently show simultaneous overexpression of $\beta$-catenin and cyclin D1. This result raises the possibility that cyclin D1 may be upregulated by $\beta$-catenin in the high-grade sarcomas previously referred to as high-grade endometrial stromal sarcoma. The underlying mechanisms responsible for nuclear $\beta$-catenin accumulation in endometrial stromal tumors and related highgrade sarcomas remain unclear.

\section{Acknowledgement}

This study was supported by a Grant-in-Aid for Scientific Research (B) (no. 21390107) from the Japan Society for the Promotion of Science, Tokyo, Japan.

\section{Disclosure/conflict of interest}

The authors declare no conflict of interest.

\section{References}

1 Hendrickson MR, Tavassoli FA, Kempson RL, et al. Mesenchymal tumours and related lesions. In: Tavassoli FA, Devilee P (eds). World Health Organization Classification of Tumours. Pathology and Genetics of Tumours of the Breast and Female Genital Organs. IARC Press: Lyon, France, 2003, pp 233-244.

2 Kurihara S, Oda Y, Ohishi Y, et al. Endometrial stromal sarcomas and related high-grade sarcomas: immunohistochemical and molecular genetic study of 31 cases. Am J Surg Pathol 2008;32:1228-1238.

3 Ilyas M. Wnt signalling and the mechanistic basis of tumour development. J Pathol 2005;205:130-144.

4 Ng TL, Gown AM, Barry TS, et al. Nuclear beta-catenin in mesenchymal tumors. Mod Pathol 2005;18:68-74.

5 Saito T, Oda Y, Sakamoto A, et al. Prognostic value of the preserved expression of the E-cadherin and catenin families of adhesion molecules and of beta-catenin mutations in synovial sarcoma. J Pathol 2000;192: 342-350.

6 Saito T, Oda Y, Tanaka K, et al. Beta-catenin nuclear expression correlates with cyclin D1 overexpression in sporadic desmoid tumours. J Pathol 2001;195:222-228.

7 Tetsu O, McCormick F. Beta-catenin regulates expression of cyclin D1 in colon carcinoma cells. Nature 1999;398:422-426.

8 Brabletz T, Jung A, Dag S, et al. $\beta$-Catenin regulates the expression of the matrix metalloproteinase-7 in human colorectal cancer. Am J Pathol 1999;155:1033-1038.

9 Matono H, Oda Y, Nakamori $\mathrm{M}$, et al. Correlation between beta-catenin nuclear expression and matrix metalloproteinase-7 overexpression in sporadic desmoid tumors. Hum Pathol 2008;39:1802-1808.

10 Deryugina EI, Quigley JP. Matrix metalloproteinases and tumor metastasis. Cancer Metastasis Rev 2006; 25:9-34.

11 Ii M, Yamamoto H, Adachi Y, et al. Role of matrix metalloproteinase-7 (matrilysin) in human cancer invasion, apoptosis, growth, and angiogenesis. Exp Biol Med 2006;231:20-27.

12 Lee AY, He B, You L, et al. Expression of the secreted frizzled-protein gene family is downregulated in human mesothelioma. Oncogene 2004;23:6672-6676.

13 He B, Lee AY, Dadfarmay S, et al. Secreted frizzledrelated protein 4 is silenced by hypermethylation and induces apoptosis in $\beta$-catenin-deficient human mesothelioma cells. Cancer Res 2005;65:743-748.

14 Liu TH, Raval A, Chen SS, et al. CpG island methylation and expression of the secreted frizzledrelated protein gene family in chronic lymphocytic leukemia. Cancer Res 2006;66:653-658.

15 Hrzenjak A, Tippl M, Kremser ML, et al. Inverse correlation of secreted frizzled-related protein 4 and beta-catenin expression in endometrial stromal sarcomas. J Pathol 2004;204:19-27.

16 Silverberg SG, Kurman RJ. Endometrial stromal tumors. In: Silverberg SG, Kurman RJ (eds). Atlas of Tumor Pathology. Tumors of the Uterine Corpus and Gestational Trophoblastic Disease. Third Series, Fascicle 3. Armed Forces Institute of Pathology: Washington, DC, 1992, pp 91-112.

17 Iwao K, Nakamori S, Kameyama M, et al. Activation of the $\beta$-catenin gene by interstitial deletions involving exon 3 in primary colorectal carcinomas without adenomatous polyposis coli mutations. Cancer Res 1998;58:1021-1026. 
18 Yagi OK, Akiyama Y, Ohkura Y, et al. Analyses of the APC and TGF-beta type II receptor genes, and microsatellite instability in mucosal colorectal carcinomas. Jpn J Cancer Res 1997;88:718-724.

19 Zou H, Molina JR, Harrington JJ, et al. Aberrant methylation of secreted frizzled-related protein genes in esophageal adenocarcinoma and Barrett's esophagus. Int J Cancer 2005;115:584-591.

20 Koontz JI, Soreng AL, Nucci M, et al. Frequent fusion of the JAZF1 and JJAZ1 genes in endometrial stromal tumors. Proc Natl Acad Sci USA 2001;98:6348-6353.

21 Ewen ME, Lamb J. The activities of cyclin D1 that drive tumorigenesis. Trends Mol Med 2004;10:158-162.

22 Wang HL, Wang J, Xiao SY, et al. Elevated protein expression of cyclin D1 and fra-1 but decreased expression of c-myc in human colorectal adenocarcinomas overexpressing $\beta$-catenin. Int J Cancer 2002;101:301-310.

23 Lin SY, Xia W, Wang JC, et al. Beta catenin, a novel prognostic marker for breast cancer: its roles in cyclin d1 expression and cancer progression. Proc Natl Acad Sci USA 2000;97:4262-4266.

24 Lantsov D, Meirmanov S, Nakashima M, et al. Cyclin D1 overexpression in thyroid papillary microcarcinoma: its association with tumour size and aberrant betacatenin expression. Histopathology 2005;47:248-256.

25 Alao J. The regulation of cyclin D1 degradation: roles in cancer development and the potential for therapeutic invention. Mol Cancer 2007;6:24.

26 Jung CK, Jung JH, Lee A, et al. Diagnostic use of nuclear $\beta$-catenin expression for the assessment of endometrial stromal tumors. Mod Pathol 2008;21:756-763.

27 Micci F, Heim S. Pathogenetic mechanisms in endometrial stromal sarcoma. Cytogenet Genome Res 2007;118:190-195.
28 Nucci MR, Harburger D, Koontz J, et al. Molecular analysis of the JAZF1-JJAZ1 gene fusion by RT-PCR and fluorescence in situ hybridization in endometrial stromal neoplasms. Am J Surg Pathol 2007;31: 65-70.

29 Oliva E, Leval L, Soslow RA, et al. High frequency of $J A Z F 1-J J A Z 1$ gene fusion in endometrial stromal tumors with smooth muscle differentiation by interphase FISH detection. Am J Surg Pathol 2007;31: 1277-1284.

30 Hrzenjak A, Moinfar F, Tavassoli FA, et al. JAZF1/ $J J A Z 1$ gene fusion in endometrial stromal sarcomas: molecular analysis by reverse transcriptase-polymerase chain reaction optimized for paraffin-embedded tissue. J Mol Diagn 2005;7:388-395.

31 Huang HY, Ladanyi M, Soslow RA. Molecular detection of JAZF1-JJAZ1 gene fusion in endometrial stromal neoplasms with classic and variant histology: evidence for genetic heterogeneity. Am J Surg Pathol 2004;28:224-232.

32 Zaloudek C, Hendrickson MR. Mesenchymal tumors of the uterus. In: Kurman RJ (ed). Blaustein's Pathology of the Female Genital Tract. 5th edn. Springer-Verlag: New York, 2002, pp 583-593.

33 Micci F, Walter CU, Teixeira MR, et al. Cytogenetic and molecular genetic analyses of endometrial stromal sarcoma: nonrandom involvement of chromosome arms $6 \mathrm{p}$ and $7 \mathrm{p}$ and confirmation of JAZF1/JJAZ1 gene fusion in $\mathrm{t}(7 ; 17)$. Cancer Genet Cytogenet 2003;144:119-124.

34 Zhu XQ, Shi YF, Cheng XD, et al. Immunohistochemical markers in differential diagnosis of endometrial stromal sarcoma and cellular leiomyoma. Gynecol Oncol 2004;92:71-79. 